Internat. J. Math. \& Math. Sci.

\title{
GRAPHS WHICH HAVE PANCYCLIC COMPLEMENTS
}

\author{
H. JOSEPH STRAIGHT \\ Department of Mathematics \\ SUNY College at Fredonia \\ Fredonia, New York 14063 \\ (Received January 23, 1978 and in revised form March
}

1978)

ABSTRACT. Let $p$ and $q$ denote the number of vertices and edges of a graph $G$, respectively. Let $\Delta(G)$ denote the maximum degree of $G$, and $\bar{G}$ the complement of G. A graph $G$ of order $p$ is said to be pancyclic if $G$ contains a cycle of each length $n, 3 \leq n \leq p$. For a nonnegative integer $k$, a connected graph $G$ is said to be of rank $\underline{k}$ if $q=p-1+k$. (For $k$ equal to 0 and 1 these graphs are called trees and unicyclic graphs, respectively.)

In 1975, I posed the following problem: Given $k$, find the smallest positive integer $p_{k}$, if it exists, such that whenever $G$ is a rank $k$ graph of order $p \leq p_{k}$ and $\Delta(G)<p-2$ then $\bar{G}$ is pancyclic. In this paper it is shown that a result by Schmeichel and Hakiml (2) guarantees that $p_{k}$ exists. It is further shown that for $k=0,1$, and $2, p_{k}=5,6$, and 7 , respectively.

1. INTRODUCTION.

Throughout this paper the terminology of Behzad and Chartrand (1) will be 
followed. In particular, $\mathrm{p}$ and $\mathrm{q}$ shall denote the number of vertices and edges of a graph $G$, respectively. We let $\Delta(G)$ denote the maximum degree of $G$ and $\bar{G}$ denote the complement of $G$.

A graph $G$ of order $P$ is called pancyclic if $G$ contains a cycle of each length $n, 3 \leq n \leq p$. For a nonnegative integer $k$, a connected graph $G$ is said to be of rank $\underline{k}$ if $q=p-1+k$. Here the number $k$ gives the number of independent cycles in $G$. When $k$ equals 0 or 1 these graphs are called trees or unicyclic graphs, respectively.

In this paper we explore the following idea: if $\mathrm{G}$ is a graph having, in some sense, little cycle structure relative to its order, then perhaps $\bar{G}$ will have a great deal of cycle structure. As an example, consider the graph shown in Figure 1. This graph is a tree, 1.e., a connected graph having no cycles. On the other hand note that its complement is pancyclic.
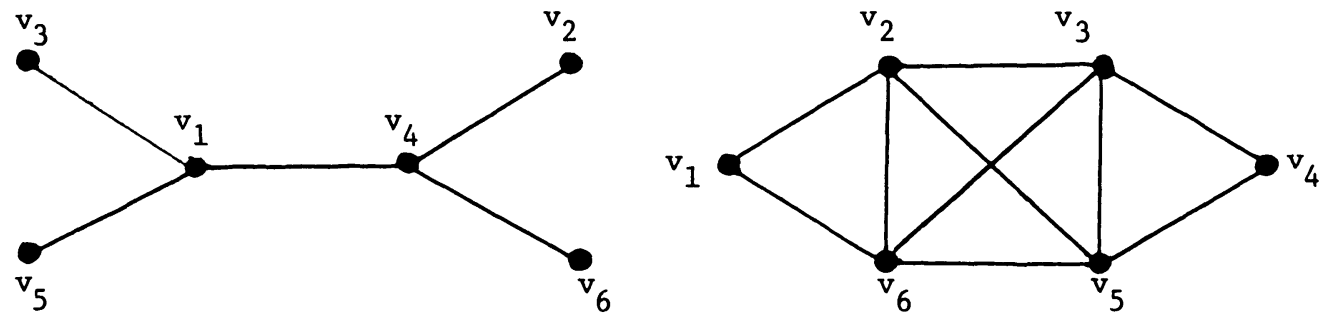

Figure 1. A tree... and its pancyclic complement

In 1975 , after obtaining the results for $k=0,1$, and 2 which are presented here, I posed the following problem: Given $k$, find the smallest positive integer $p_{k}$, if it exists, such that whenever $G$ is a graph of rank $k$ of order $p \geq p_{k}$ and $\Delta(G)<p-2$, then $\bar{G}$ is pancyclic. Recently, J. A. Bondy has pointed out that the existence of $\mathrm{p}_{k}$ is guaranteed by the following result due to Schmeichel and Hakimi [2]. 
THEOREM. Let $G$ be a graph with $p$ vertices, $q$ edges, and minimum degree $\delta \geq 2$. If

$$
q> \begin{cases}\frac{1}{2}\left(p^{2}-(2 \delta+1) p+3 \delta^{2}+\delta\right), & 2 \leq \delta \leq \frac{p+5}{6} \text { and } p \text { odd } \\ \frac{1}{2}\left(p^{2}-(2 \delta+1) p+3 \delta^{2}+\delta\right), & 2 \leq \delta \leq \frac{p+8}{6} \text { and } p \text { even } \\ \frac{1}{8}\left(3 p^{2}-8 p+5\right)+\delta, & \frac{p+5}{6} \leq \delta \leq \frac{p-1}{2} \text { and } p \text { odd } \\ \frac{1}{8}\left(3 p^{2}-10 p+16\right)+\delta, & \frac{p+8}{6} \leq \delta \leq \frac{p-2}{2} \text { and } p \text { even } \\ \frac{1}{4} p^{2}, & \frac{p-1}{2}<\delta\end{cases}
$$

then $G$ is pancyclic.

COROLLARY. Let $k$ be a nonnegative integer. Then there exists a positive integer $p_{k}$ such that whenever $G$ is a graph of rank $k$ of order $\mathrm{p} \geq \mathrm{p}_{\mathrm{k}}$ and $\Delta(G)<\mathrm{p}-2$, then $\bar{G}$ is pancyclic.

PROOF. Let $G$ be a graph of rank $k$ with $\Delta(G)<p-2$. If $G$ has $p$ vertices, then $\bar{G}$ has $p$ vertices, $q=\frac{p^{2}-3 p+2}{2}-k$ edges and minimum degree $\delta \geq 2$. Depending on the value of $\delta$, the requirements for $q$ given by the theorem would yield the following inequalities:

$$
\begin{aligned}
& (2 \delta-2) \mathrm{p}>3 \delta^{2}+\delta+2 \mathrm{k}-2 \\
& \mathrm{p}^{2}-4 \mathrm{p}>8 \mathrm{k}+8 \delta-3 \\
& \mathrm{p}^{2}-2 \mathrm{p}>8 \mathrm{k}+8 \delta+8 \\
& \mathrm{p}^{2}-6 \mathrm{p}>4 \mathrm{k}-4
\end{aligned}
$$

Note that each of the above inequalities is true provided that $p$ is large enough. Hence we can choose $p_{k}$ to be the least positive integer which makes all the above inequalities true.

The above theorem yields an upper bound for $\mathrm{p}_{k}$; however, in the known cases it does not give us a very good bound. For example, the theorem 
would tell us that $p_{2} \leq 10$, whereas we will show that $p_{2}=7$. In the remainder of the paper we show that for $k=0,1$, and $2, p_{k}=5,6$, and 7 , respectively.

\section{MAIN RESULTS.}

THEOREM 1. If $\mathrm{G}$ is a tree of order $\mathrm{p} \geq 5$ with $\Delta(G)<\mathrm{p}-2$, then $\bar{G}$ is pancyclic.

PROOF. The proof is by induction on $p$. If $p=5$, then $G$ is a path on 5 vertices. Thus $\bar{G}=C_{5}+e$ and so clearly $\bar{G}$ is pancyclic. Now let $p \geq 6$, and assume the result holds for all trees of order less than $p$. Let $\mathrm{G}$ be a tree of order $\mathrm{p}$ with $\Delta(G)<\mathrm{p}-2$. If $\Delta(G)<\mathrm{p}-3$, let $\mathrm{v}$ be an end vertex of $G$. If $\Delta(G)=p-3$, then unless $G$ is the graph of Figure 1, we may choose $v$ to be an end vertex adjacent with the unique vertex of degree $p-3$. Now consider $G-v$, which is a tree of order $\mathrm{p}-1$ with $\Delta(G-\mathrm{v})<(\mathrm{p}-1)-2$. Hence by the induction hypothesis, $\overline{G-v}$ has a cycle of each length $n, 3 \leq n \leq p-1$. Therefore so does $\bar{G}$. Since $\operatorname{deg}_{G} v=p-2>\frac{p-1}{2}, v$ must be adjacent in $\bar{G}$ to two consecutive vertices on the $(p-1)-c y c l e$ in $\overline{G-v}$. Thus this cycle can be extended in $\bar{G}$ to a cycle of length $p$. Therefore, $\bar{G}$ is pancyclic. Now by induction the proof is completed.

COROLLARY 1. If $\mathrm{G}$ is a forest of order $\mathrm{p} \geq 5$ with $\Delta(G)<\mathrm{p}-2$, then $\bar{G}$ is pancyclic.

PROOF. Note that there exists a tree $\mathrm{H}$ with $\Delta(\mathrm{H})<\mathrm{p}-2$ containing $G$ as a spanning subgraph. Since $\bar{H}$ is pancyclic and $\bar{H} \subseteq \bar{G}, \bar{G}$ is pancyclic.

THEOREM 2. If $G$ is a unicyclic graph of order $p \geq 6$ with $\Delta(G)<p-2$, then $\bar{G}$ is pancyclic.

PROOF. Let $u_{1}, u_{2}, \ldots, u_{n}$ denote the cycle vertices of $G$. Among the vertices $u_{1}$, we will choose one of minimum degree in $G$; call it $u$. 
CASE 1. Suppose $n \geq 4$. Then $\operatorname{deg} u \leq 2+\frac{p-4}{4}=\frac{p+4}{4}$. Note that $\frac{p+4}{4}<\frac{p-1}{2}$ provided that $\mathrm{p} \geq 6$. If $\Delta(G)=p-3$, notice that we can choose u so that $\Delta(G-u)=p-4$ unless $G$ is the graph of Figure 3a. Now since $G-u$ is a forest, $\overline{G-u}$ is pancyclic by Corollary 1 . Since $u$ is adjacent in $\bar{G}$ to two consecutive vertices on the $(p-1)$-cycle of $\overline{G-u}$, this cycle can be extended to a p-cycle in $\bar{G}$. Therefore, $\bar{G}$ is pancyclic.

CASE 2. Suppose $n=3$. First, suppose that if $\Delta(G)=p-3=\operatorname{deg} v$, then $\mathrm{v}$ is one of the 3 cycle vertices. Then we know that $\Delta(G-u) \leq p-4$. Now $\operatorname{deg} u \leq 2+\frac{p-3}{3}=\frac{p+3}{3}$. If $\operatorname{deg} u<\frac{p-1}{2}$ we can proceed just as in Case 1. This will happen if $p>9$ or if $p=8$. If it does not happen then $G$ must be one of the graphs shown in Figures $3 b-d$, $a 11$ of which have pancyclic complements. Secondly suppose that $\Delta(G)=p-3=\operatorname{deg}_{G} v$ and $v$ is not a cycle vertex. Then $G$ is the graph of Figure 2 .

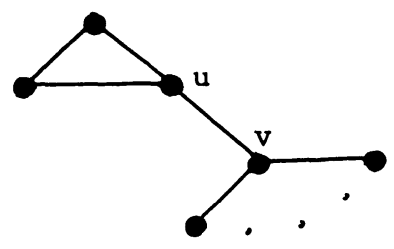

Figure 2

If $p \geq 8$, we can remove $u$ and proceed as above. If $p=6$ or 7 , we again get special case graphs, which can be shown to have pancyclic complements. 


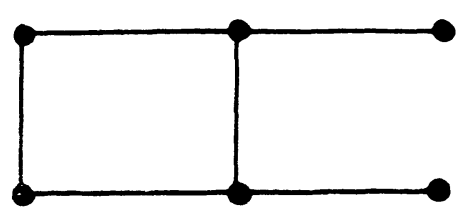

a.

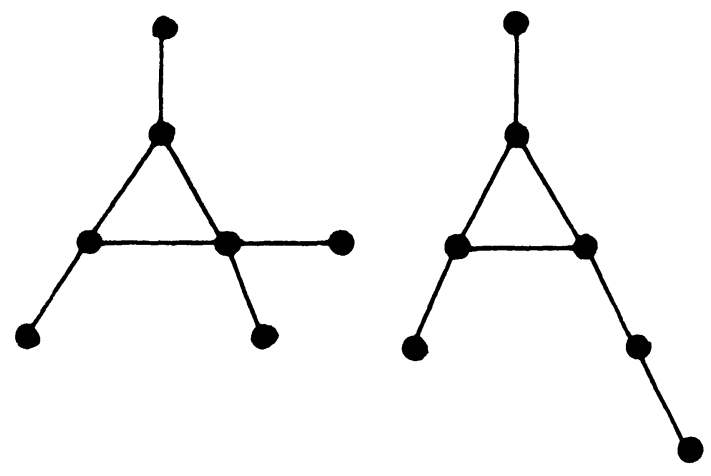

c. $p=7$

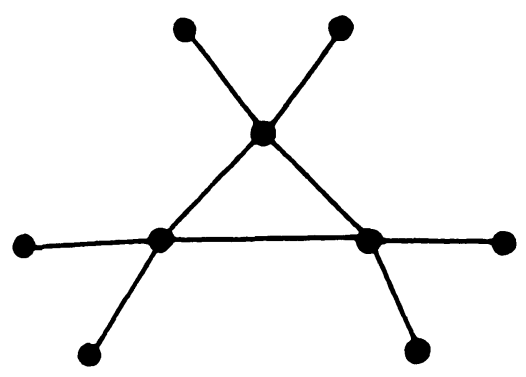

b. $p=9$

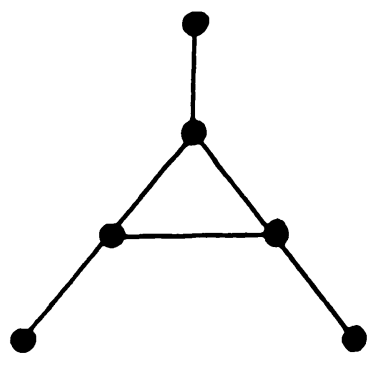

d. $p=6$

\section{Figure 3}

COROLLARY 2. If $G$ is a graph of order $P \geq 6$ with $\Delta(G)<p-2$ and G contains exactly one cycle, then $\bar{G}$ is pancyclic.

The five-cycle $\mathrm{C}_{5}$ is a unicyclic graph on 5 vertices which does not have a pancyclic complement. This shows that $p_{1}=6$. The graph shown in Figure 4 is a rank 2 graph of order 6 whose complement does not contain a 3-cycle. Hence $p_{2} \geq 7$. Our final result shows that indeed $p_{2}=7$. 


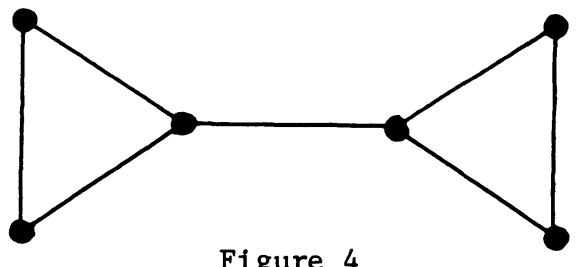

Figure 4

THEOREM 3. If $G$ is a graph of rank 2 of order $p \geq 7$ with $\Delta(G)<p-2$, then $\bar{G}$ is pancyclic.

PROOF. We consider three cases.

CASE 1. G has a cycle with a diagonal. Let $u_{1}, u_{2}, \ldots, u_{n}$ be the cycle vertices of $G, n \geq 4$, and suppose $u_{1} u_{i}$ is the diagonal of the cycle, $3 \leq 1 \leq n-1$. First, suppose $\Delta(G)<p-3$. Choose a cycle vertex $u$ which has the smallest degree in $G$ among the cycle vertices. Then $\operatorname{deg} u \leq \frac{p+6}{4}$. Note that $\frac{p+6}{4}<\left\{\frac{p-1}{2}\right\}$ if $p \geq 8$. Also there does not exist a rank 2 graph on 7 vertices with $\Delta(G)=3$ and $\operatorname{deg} u_{i}=3,1 \leq 1 \leq n$. Thus $\mathrm{p} \geq 7$ implies $\operatorname{deg}_{\mathrm{G}} \mathrm{u}<\left\{\frac{\mathrm{p}-1}{2}\right\}$. Now by Corollary $2, \overline{\mathrm{G}-\mathrm{u}}$ is pancyclic. Since $u$ is adjacent in $\bar{G}$ to more than half the other vertices, $\bar{G}$ is pancyclic. Secondly, suppose $\Delta(G)=p-3$ and $p \geq 8$. Then we either choose $u$ as above or of degree 3 in $G$ in such a way that $\Delta(G-u)=p-4$. Since $3<\frac{p-1}{2}, \operatorname{deg}_{G} u<\frac{p-1}{2}$ and so we argue as before. Lastly we must consider the case where $p=7, \Delta(G)=4$, and there does not exist a vertex $\mathrm{u}$ with $\operatorname{deg}_{G} \mathrm{u}=2$ and $\Delta(G-u)=3$. In this case $G$ must be one of the graphs shown in Figure 5, all of which have pancyclic complements. 

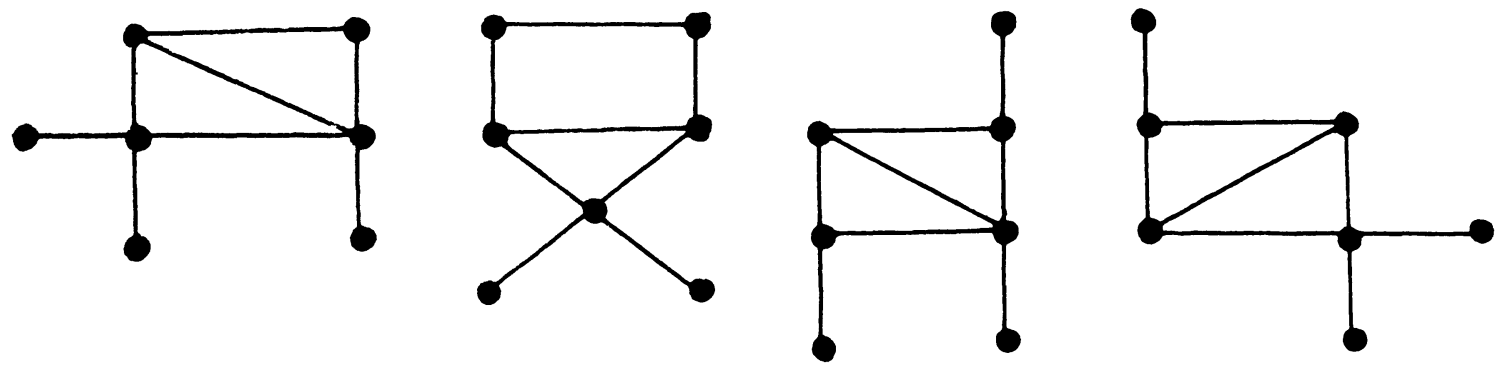

Figure 5

CASE 2. G has the following configuration as a subgraph.

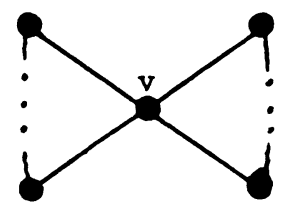

Again let $u$ be a cycle vertex of smallest degree. Then

$$
\operatorname{deg}_{G} u \leq\left\{\begin{array}{l}
2+\frac{p-3}{5}, u \neq v \\
4+\frac{p-13}{5}, u=v
\end{array} \quad=\frac{p+7}{5}<\frac{p-1}{2} .\right.
$$

Also if $\Delta(G)=p-3$, then clearly it is possible to choose u so that $\Delta(G-u)=p-4$. Hence we may argue as before.

CASE 3. G contains the configuration of Figure 6 .

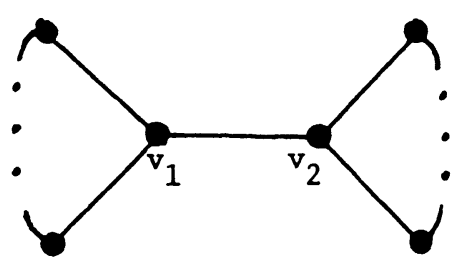

Figure 6 
Choose $u$ as before. Then

$$
\operatorname{deg}_{G} u \leq\left\{\begin{array}{l}
2+\frac{p-4}{6}, u \in\left\{v_{1}, v_{2}\right\} \\
3+\frac{p-10}{6}, u \in\left\{v_{1}, v_{2}\right\}
\end{array}=\frac{p+8}{6}<\frac{p-1}{2}\right.
$$

If $\Delta(G)=p-3$, then $\operatorname{deg} v_{1}=p-3$ for $1=1$ or 2 but not both, and all other cycle vertices have degree 2. Hence we may choose u so that $\Delta(G-u)=p-4$ and proceed as before.

\section{REFERENCES}

1. Behzad, M. and G. Chartrand. Introduction to the Theory of Graphs, Allyn and Bacon, Boston, 1971.

2. Schmeichel, E. F. and S. L. Hakimi. Pancyclic Graphs and a Conjecture of Bonday and Chvatal, J. Combinatorial Theory (B) 17 (1974) 22-34.

KEY WORDS AND PHRASES. Graphs, pancyclic graphs, and unicyclic graphs. 


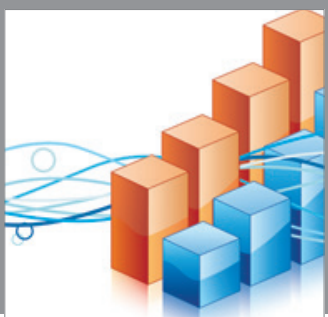

Advances in

Operations Research

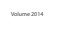

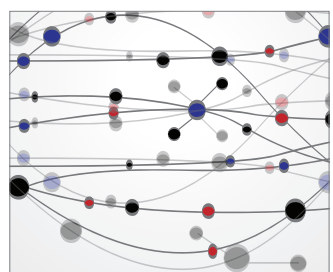

\section{The Scientific} World Journal
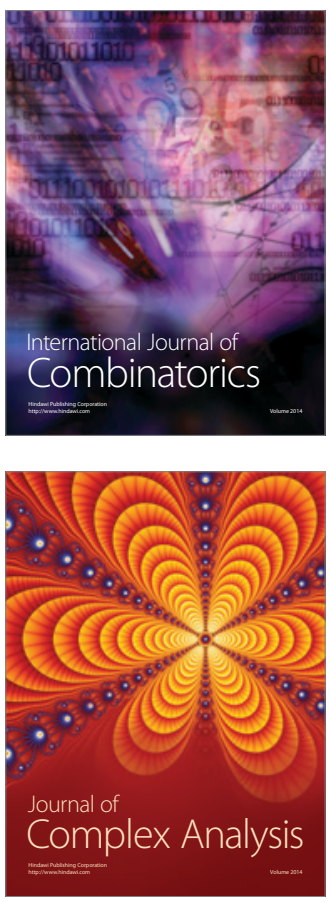

International Journal of

Mathematics and

Mathematical

Sciences
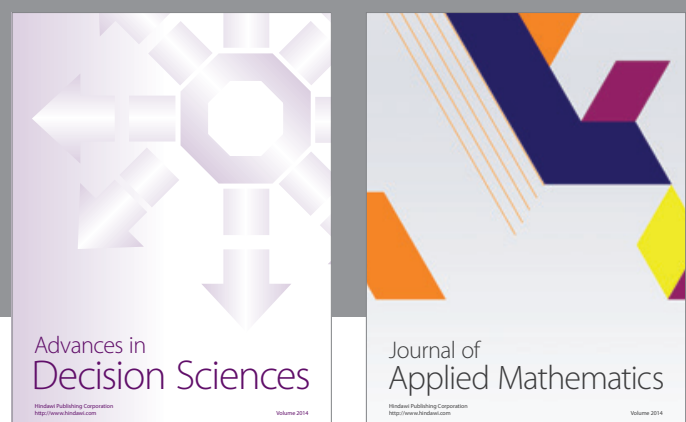

Journal of

Applied Mathematics
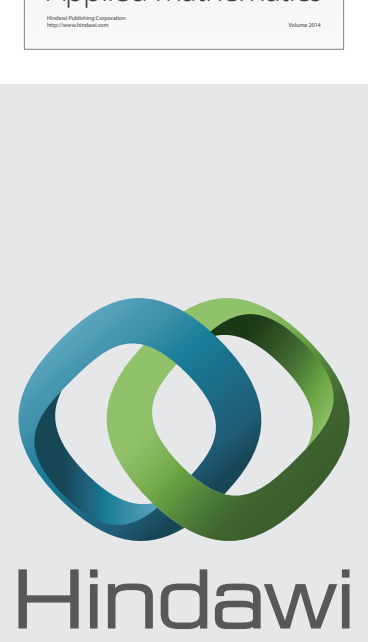

Submit your manuscripts at http://www.hindawi.com
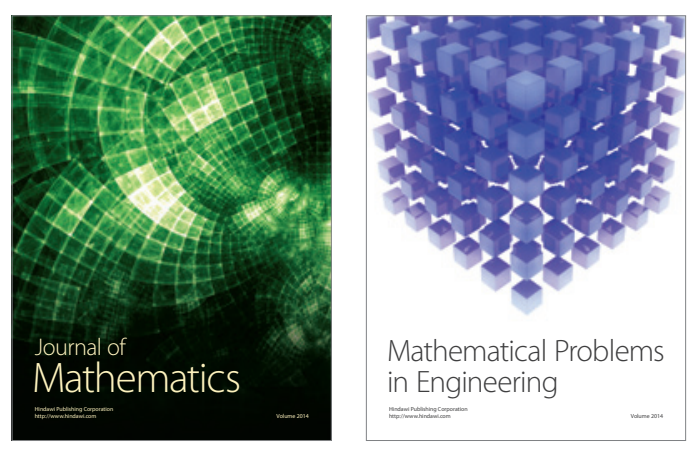

Mathematical Problems in Engineering
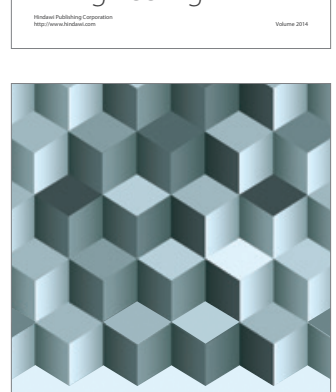

Journal of

Function Spaces
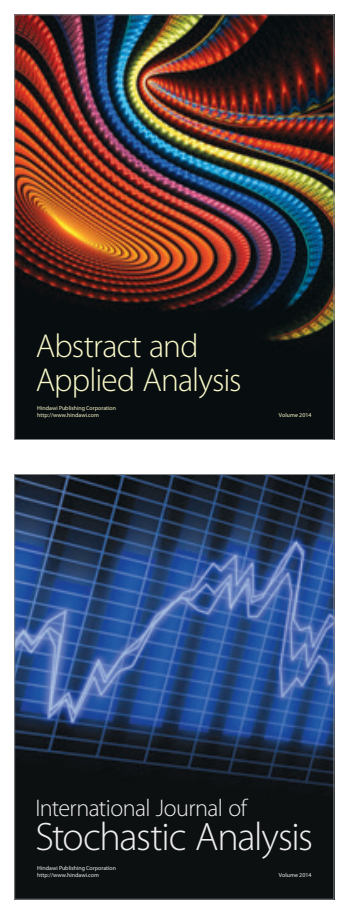

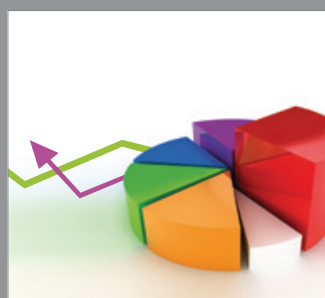

ournal of

Probability and Statistics

Promensencen
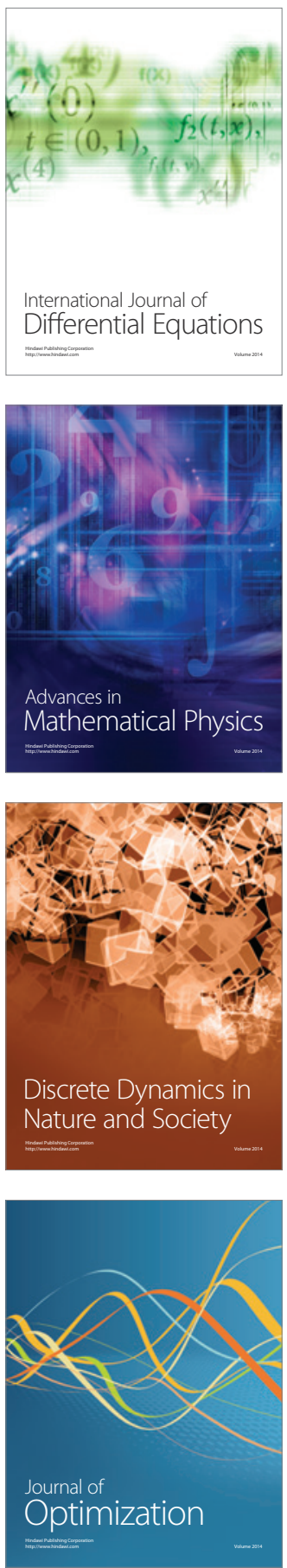